\title{
Advanced Imaging in Cardiac Sarcoidosis
}

\author{
Roberto Ramirez, Maria Trivieri, Zahi A. Fayad, Amir Ahmadi, Jagat Narula, and Edgar Argulian \\ Division of Cardiology, Icahn School of Medicine at Mount Sinai, New York, New York
}

Learning Objectives: On successful completion of this activity, participants should be able to (1) identify high-risk clinical features that necessitate evaluation for cardiac sarcoidosis; (2) understand key histopathologic features of cardiac sarcoidosis and how they relate to different imaging modalities; and (3) recognize the importance of cardiac imaging modalities in the evaluation of patients with suspected or established cardiac sarcoidosis.

Financial Disclosure: The authors of this article have indicated no relevant relationships that could be perceived as a real or apparent conflict of interest.

CME Credit: SNMMI is accredited by the Accreditation Council for Continuing Medical Education (ACCME) to sponsor continuing education for physicians. SNMMI designates each JNM continuing education article for a maximum of 2.0 AMA PRA Category 1 Credits. Physicians should claim only credit commensurate with the extent of their participation in the activity. For CE credit, SAM, and other credit types, participants can access this activity through the SNMMI website (http://www.snmmilearningcenter.org) through July 2022.

Sarcoidosis is a chronic disease of unknown etiology characterized by the presence of noncaseating granulomas. Cardiac involvement in sarcoidosis may lead to adverse outcomes such as advanced heart block, arrhythmias, cardiomyopathy, or death. Cardiac sarcoidosis can occur in patients with established sarcoidosis, or it can be the sole manifestation of the disease. Traditional diagnostic techniques, including echocardiography, have poor sensitivity for diagnosing cardiac sarcoidosis. The accumulating evidence supports the essential role of advanced cardiac imaging modalities such as MRI and PET in diagnosis, risk stratification, and management of patients with cardiac sarcoidosis. The current review highlights important theoretic and practical aspects of using cardiac imaging tools in the evaluation of patients with suspected or established cardiac sarcoidosis.

Key Words: MRI; PET; arrhythmias; cardiac sarcoidosis; cardiomyopathy; heart block

J Nucl Med 2019; 60:892-898

DOI: 10.2967/jnumed.119.228130

\section{$\mathbf{S}$}

rcoidosis is a multisystem disease that can involve the heart, among other organs (1). The prevalence of cardiac sarcoidosis (CS) has not been precisely estimated, but it is likely underrecognized when compared with autopsy series (2). Only a minority of patients with systemic sarcoidosis have clinical manifestations suggestive of cardiac involvement, yet it is important to diagnose CS because of the high incidence of (potentially preventable) electrical abnormalities and sudden cardiac death (3).

Sarcoidosis can additionally present with isolated cardiac involvement without other organs being affected. Although this presentation is rare, a recent autopsy study showed that up to $40 \%$ of patients who died suddenly from CS had no obvious extracardiac manifestations (4). Therefore, cardiac evaluation should be sought in high-risk patients, particularly in young and middle-aged patients presenting with myocardial disease, unexplained ventricular

Received Mar. 4, 2019; revision accepted Jun. 3, 2019.

For correspondence contact: Edgar Argulian, Division of Cardiology, Mt. Sinai St. Luke's Hospital, Icahn School of Medicine, 1111 Amsterdam Ave., New York, NY 10025.

E-mail: edgar.argulian@mountsinai.org

Published online Jun. 6, 2019.

COPYRIGHT (c) 2019 by the Society of Nuclear Medicine and Molecular Imaging. tachycardia, or high-degree atrioventricular block. The diagnosis of CS is hindered by the lack of any reliable biomarker or diagnostic test; shortcomings of commonly used cardiac tests (noninvasive and invasive) such as electrocardiography, echocardiography, myocardial perfusion imaging, and even endomyocardial biopsy include low sensitivity and specificity (5). The gold standard criteria used in most studies of CS have significant limitations. The Japanese Ministry of Health and Welfare criteria, originally published in 1993 and updated in 2006, have not been extensively validated (6). These criteria require either histologic confirmation of cardiac involvement via endomyocardial biopsy or clinical confirmation via a combination of major and minor criteria $(7,8)$. More recently, the Heart Rhythm Society published an expert consensus statement that provides more contemporary criteria for diagnosis of CS that include advanced cardiac imaging techniques. However, these criteria recommend tissue diagnosis of extracardiac sarcoidosis (9).

Advanced imaging modalities, that is, cardiac MR (CMR) and PET, have been shown to detect cardiac involvement with a prevalence similar to that seen in autopsy studies, and they offer prognostic value beyond traditional clinical criteria (10-13). $\mathrm{Nu}-$ merous reports have highlighted the potential benefits of advanced imaging modalities in improving the ability to identify and treat patients with CS. In modern practice, these imaging tools play a fundamental role in early diagnosis, assessment of disease activity, prognostication, and monitoring of therapeutic response.

\section{PATHOLOGY RELEVANT FOR CARDIAC IMAGING}

It is important to understand certain histopathologic features of $\mathrm{CS}$ as they relate to different imaging modalities. The myocardium is most frequently involved in CS; the pericardium and endocardium are less frequently affected, and their involvement typically results from direct extension of myocardial disease. The pathologic description of sarcoidosis includes 3 histologic stages: edema, granulomatous infiltration, and fibrosis. Mononuclear, predominantly lymphocytic infiltration and interstitial edema are seen at early stages. The most characteristic lesions of CS are discrete, compact, nonnecrotizing, epithelioid granulomas along with areas of patchy fibrosis (14). At later stages, there is a shift from mononuclear phagocytes and CD4+ cells with a T-helper type 1 response to a T-helper type 2 response eliciting antiinflammatory effects and resulting in tissue scarring and replacement fibrosis (15). Areas of focal myocardial involvement disrupt normal myocardial electrical 
properties, predisposing to ventricular arrhythmias including malignant rhythms and sudden cardiac death. From an imaging perspective, identification of inflammation is possible because of the avidity of mononuclear inflammatory cells for ${ }^{18} \mathrm{~F}-\mathrm{FDG}$ and tissue edema as seen on T2-weighted CMR imaging. Areas of fibrosis can be identified by late gadolinium enhancement (LGE) on delayed CMR imaging. The progression of CS has not been well studied, but the natural history of focal myocardial disease can be variable, ranging from complete resolution to dense transmural fibrosis.

Pathology-proven CS most commonly affects the interventricular septum and inferior wall of the left ventricle; less commonly affected is the anterior wall of the left ventricle and right ventricle (16). Involvement of the interventricular septum accounts for the high rates of atrioventricular conduction abnormalities observed in these patients. From an imaging perspective, patients with advanced sarcoidosis have shown thinning of the basal septum, which can be appreciated on echocardiography.

In addition to a patchy distribution, lesions of CS have a known predilection for the subepicardial and midwall myocardium $(7,8)$. This contrasts with coronary artery disease, which initially affects the subendocardial region in a predictable fashion. Since the subendocardial myocardium contributes disproportionately to left ventricular regional wall motion, myocardial involvement due to coronary artery disease can be identified by wall motion analysis using echocardiography or cine CMR. On the other end, significant myocardial disease can be present in the subepicardial and midwall regions in CS, with preserved wall motion and left ventricular emptying. Heart failure is a less common initial presentation of CS, typically signifying advanced disease. Not surprisingly, modalities relying on wall motion analysis have poor sensitivity for CS. Finally, patchy involvement and the typical subepicardial or midwall distribution of CS lesions markedly decrease the sensitivity of blind endomyocardial biopsy for CS $(<25 \%)$. Electroanatomic mapping or imaging-guided procedures may increase the sensitivity of endomyocardial biopsy, but further validation of these approaches is needed (17). As a result, contemporary diagnosis and management of CS heavily rely on advanced cardiac imaging as an emerging clinical standard.

\section{DIAGNOSIS}

\section{Echocardiography}

Transthoracic echocardiography is the initial imaging modality in patients with suspected CS. It is a useful test for assessing the overall left ventricular systolic function, left ventricular geometry, areas of myocardial thickening or thinning, diastolic parameters, and right ventricular performance. Commonly described echocardiographic findings include regional wall motion abnormalities, aneurysms, thinning of the basal septum, dilation of the left ventricle, and impaired right or left ventricular systolic or diastolic function (18). At the same time, echocardiography is an insensitive technique for detection of CS, and normal transthoracic echocardiography findings cannot be used to rule out the presence of CS. Echocardiography findings can be quite specific in sarcoidosis patients with cardiac symptoms or abnormal electrocardiography findings. Abnormal echocardiography findings in these patients is highly suggestive of CS, with a positive predictive value of up to $92 \%$ (19).

Basal interventricular septal thinning has been described as a characteristic finding in patients with CS (Fig. 1). In one study, interventricular septal thinning (defined as a basal interventricular septum thickness $\leq 4 \mathrm{~mm}$ or a basal interventricular septum-to-

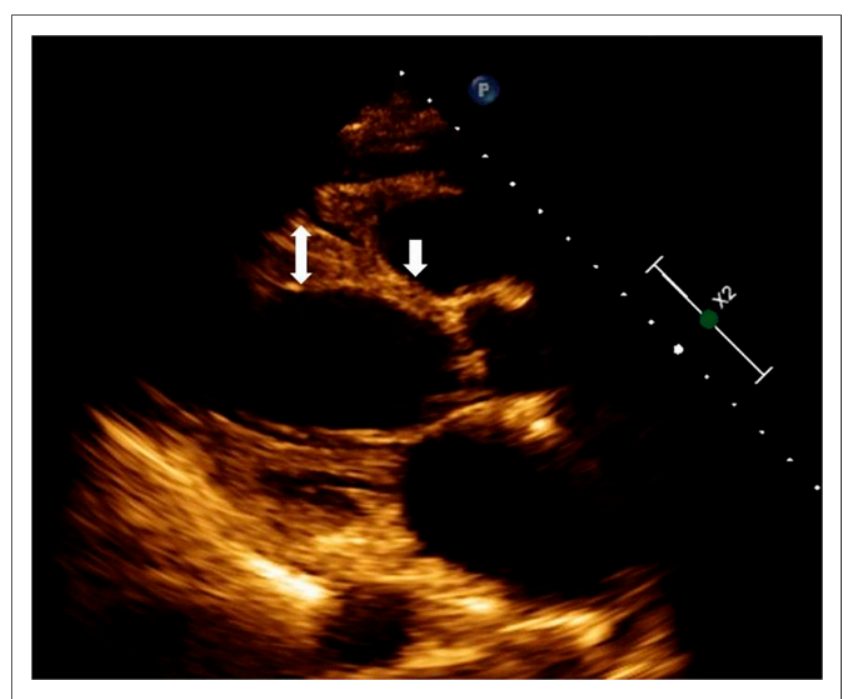

FIGURE 1. Thinning of basal septum in patient with CS as seen on transthoracic echocardiography. Thinning (arrow) is obvious when compared with mid anteroseptum (double-head arrow).

interventricular septum ratio $\leq 0.6$ at the time of CS diagnosis) was associated with poor long-term clinical outcomes $(20)$.

Because of comorbid lung disease, patients with sarcoidosis may have elevated right heart pressures. Sarcoidosis-related pulmonary hypertension is an uncommon complication. In a recent large cohort with biopsy-confirmed sarcoidosis, the prevalence of pulmonary hypertension was estimated at $12 \%(21)$, and it was associated with increased morbidity and mortality (22).

Speckle-tracking echocardiography has been introduced as a new echocardiographic technique to assess regional and global left ventricular strain and has shown promise in early diagnosis of CS. It is helpful for the detection of early changes in myocardial mechanics before left ventricular systolic dysfunction becomes apparent. Joyce et al. evaluated 100 patients with systemic sarcoidosis without known CS or other heart disease. When compared with controls, patients with sarcoidosis had impaired global longitudinal strain, and the strain abnormality was associated with a higher rate of major adverse cardiovascular events (23). These findings suggest the need for larger prospective studies to assess the accuracy of this technique in early diagnosis of CS.

\section{SPECT}

Myocardial perfusion imaging using ${ }^{201} \mathrm{Tl}$ and ${ }^{99 \mathrm{~m}} \mathrm{Tc}-$ based SPECT can identify focal perfusion defects at rest, with either a fixed or a reverse redistribution pattern with vasodilator stress (24-27). Resting myocardial perfusion defects correspond to microvascular compression or fibrogranulomatous replacement of myocardium. Although these defects may not follow the pattern typical for coronary artery disease, alternative diagnoses, specifically coronary artery disease, should be ruled out before attributing abnormalities to CS. When stress testing is performed, these defects may improve on stress imaging (in contrast to coronary artery disease). This finding is referred to as reverse distribution, which is believed to be secondary to focal reversible microvascular constriction in coronary arterioles around granulomas, although this phenomenon is not specific to CS $(28,29) .{ }^{201} \mathrm{Tl}$ and ${ }^{99 \mathrm{~m}} \mathrm{Tc}$-based SPECT myocardial perfusion imaging can be used with ${ }^{18} \mathrm{~F}-\mathrm{FDG}$ PET for combined assessment of perfusion and inflammation only if appropriate attenuation correction is available. Most centers prefer 
to use PET because of greater ease of interpreting 2 sets of images acquired using the same modality, the robust attenuation correction of PET, and the generally higher spatial resolution of PET than of SPECT. Gallium scintigraphy has been used to detect active inflammatory disease, but it has been abandoned by most centers in the United States because of poor sensitivity and low spatial resolution.

\section{${ }^{18}$ F-FDG PET}

${ }^{18} \mathrm{~F}-\mathrm{FDG}$ is a glucose analog that is useful in detecting active CS. The ability of ${ }^{18}$ F-FDG PET to image inflammation in sarcoidosis is due to an increased uptake of ${ }^{18} \mathrm{~F}$-FDG in macrophage-dense regions. Both glucose and ${ }^{18} \mathrm{~F}$-FDG become phosphorylated in active macrophages; while glucose is further metabolized, ${ }^{18} \mathrm{~F}-\mathrm{FDG}$ phosphate remains in macrophages and can be imaged. Inflammation imaging is typically combined with resting perfusion assessment using PET myocardial perfusion imaging (with ${ }^{13} \mathrm{~N}$-ammonia or ${ }^{82} \mathrm{Rb}$ ) or SPECT. Perfusion defects may be seen by PET or SPECT in the presence of inflammation because of compression of the microvasculature or fibrosis leading to a mismatch between perfusion and ${ }^{18} \mathrm{~F}$-FDG metabolism. Combining inflammation and perfusion imaging permits assessment of the full spectrum of CS and provides valuable diagnostic and prognostic information. The presence of multiple areas of uptake combined with matched perfusion abnormalities makes the diagnosis very likely. At the same time, regional ${ }^{18} \mathrm{~F}-\mathrm{FDG}$ uptake by itself is not specific to CS. As an example, isolated low-intensity lateral-wall ${ }^{18} \mathrm{~F}$-FDG uptake without perfusion abnormalities has a lower probability of being diagnostic. Similarly, high ${ }^{18} \mathrm{~F}$-FDG uptake can be seen in hibernating myocardium because of chronic ischemia in patients with coronary artery disease, as well as in cardiomyopathies with an inflammatory component such as active myocarditis or systemic rheumatologic conditions with cardiac involvement. Diffuse ${ }^{18}$ F-FDG uptake may be seen in patients who have undergone inadequate preparation for the test. On the other hand, resting perfusion defects may be present in patients with CS that has no significant inflammatory component. Therefore, the absence of ${ }^{18} \mathrm{~F}$-FDG uptake should be interpreted as a sign of no active myocardial inflammation but cannot rule out the presence of CS (30). Staging systems have been proposed for CS using the combination of inflammation and perfusion radionuclide imaging, but these classifications lack histologic or outcome validation (31).

PET as a diagnostic modality for CS has few advantages. It can be safely performed on patients with intracardiac devices and advanced renal disease. Another advantage of PET with wholebody imaging is the ability to evaluate extracardiac sarcoidosis. The lungs are the most common site of involvement, and the thoracic lymph nodes are frequently affected, showing bilateral hilar and mediastinal lymphadenopathy on PET imaging (1).

Several studies have attempted to determine the accuracy of cardiac PET for diagnosing CS, but the true diagnostic performance is largely unknown. In a metaanalysis by Youssef et al. (32) that included 164 patients, the collected data showed a pooled sensitivity of $89 \%$ and a pooled specificity of $78 \%$ in diagnosing CS. In a more recent metaanalysis of 17 studies, the pooled sensitivity of PET was $84 \%$ and the pooled specificity was $83 \%$ (33). However, these findings should be interpreted with caution because of the limitations of the gold standard itself and the pooling of small studies.

The diagnostic performance of ${ }^{18} \mathrm{~F}$-FDG PET relies on the appropriate suppression of physiologic glucose utilization by normal cardiomyocytes. To improve specificity in identifying pathologic glucose uptake, several methods have been proposed (although none has been standardized for CS), including prolonged fasting, dietary manipulation with a high-fat, very low carbohydrate diet, intravenous heparin, and often a combination of these approaches (30). These strategies may be ineffective in up to $25 \%$ of the patients, leading to potentially false-positive or inconclusive results (34). Because of these limitations, several studies have evaluated alternative tracers with higher specificity for inflammatory or proliferating cells and without the inconvenience of complicated dietary or fasting preparations. A study by Norikane et al. (35) compared the diagnostic accuracy of $3^{\prime}$-deoxy-3'- ${ }^{18} \mathrm{~F}$-fluorothymidine and ${ }^{18} \mathrm{~F}$ FDG in patients with newly diagnosed cardiac or extracardiac sarcoidosis. The study showed that $3^{\prime}$-deoxy $-3^{\prime}-{ }^{18} \mathrm{~F}$-fluorothymidine uptake in sarcoid lesions was significantly lower than ${ }^{18} \mathrm{~F}$ FDG uptake, although sensitivity and specificity (92\% and $100 \%$, respectively) did not significantly differ between the 2 tracers. Alternative tracers that bind to somatostatin receptors on inflammatory cells in sarcoid granulomas, such as ${ }^{68} \mathrm{Ga}$-DOTANOC, may decrease the proportion of inconclusive studies (36). Gormsen et al. (37) compared the diagnostic accuracy and interobserver variability of ${ }^{68} \mathrm{Ga}$-DOTANOC with ${ }^{18} \mathrm{~F}$-FDG PET. The study showed that ${ }^{68} \mathrm{Ga}$-DOTANOC has a higher diagnostic accuracy $(100 \%$ vs. $79 \%$ ) and a lower interobserver variability, although the study population was small. Neither $3^{\prime}$-deoxy-3'-18 F-fluorothymidine nor ${ }^{68} \mathrm{Ga}$-DOTANOC requires fasting or dietary restrictions.

\section{CMR}

CMR is an important advanced imaging modality to screen or evaluate patients with CS, since it allows detection of myocardial edema, perfusion abnormalities, and scarring. It also allows detailed assessment of biventricular geometry and function. Reportedly, CMR has a high sensitivity and specificity for diagnosis of CS (sensitivity, 75\%-100\%; specificity, 76\%-78\%) (38,39). In addition, CMR is useful for identifying areas for endomyocardial biopsy and increasing the sensitivity of tissue diagnosis.

CMR can also potentially evaluate the inflammatory component of CS. CMR is able to detect edema and inflammation with the addition of T2-weighted imaging and T2 mapping. Although T2weighted CMR has been suggested as a potential alternative to ${ }^{18} \mathrm{~F}-\mathrm{FDG}$ PET in detecting inflammation and monitoring response to therapy, this technique suffers from a relatively low signal-tonoise ratio and needs further clinical validation $(40,41)$.

LGE on delayed imaging is used for evaluation of myocardial scarring (Fig. 2). Gadolinium, an extracellular contrast agent, demonstrates slow washout from areas of fibrosis and inflammation relative to normal myocardium. Although various patterns of LGE may be seen, sarcoidosis lesions are commonly localized in the septal, basal, and lateral segments of the left ventricle and papillary muscles, with relative sparing of the subendocardium $(39,42)$. The LGE distribution and the overall LGE pattern are helpful in recognizing CS but may also be nonspecific. The extent of LGE can be quantified; however, there is currently no consensus on direct quantification for CS diagnosis. In addition, CMR imaging is limited in patients with cardiac pacemakers or implantable cardioverter-defibrillator devices, and the use of gadolinium is contraindicated in patients with advanced renal disease.

\section{THERAPEUTIC AND PROGNOSTIC CONSIDERATIONS OF CARDIAC PET AND CMR}

\section{${ }^{18}$ F-FDG PET}

Emerging data support the role of ${ }^{18} \mathrm{~F}-\mathrm{FDG}$ PET in prognosticating patients with CS. A higher risk of ventricular arrhythmias and death has been described in patients with ${ }^{18} \mathrm{~F}$-FDG uptake and 


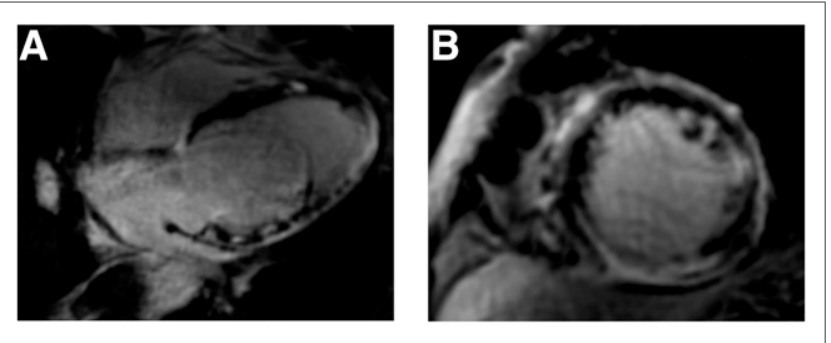

FIGURE 2. CMR findings in patient with CS. Four-chamber (A) and short-axis (B) views demonstrate extensive, patchy areas of LGE throughout myocardium.

focal perfusion defects. In one study of 118 patients with no known coronary artery disease referred for cardiac PET because of established or suspected CS, those with both myocardial perfusion defects and abnormal ${ }^{18}$ F-FDG uptake had a 4-fold increase in the annual rate of ventricular tachycardia and death. Although inflammation of the right ventricle was rare, those with focal right ventricular inflammation had a 5-fold higher event rate than those with normal perfusion and metabolism. On the other hand, the presence or absence of active extracardiac sarcoidosis was not associated with adverse events (13). A similar finding was described by Tuominen et al. in a retrospective analysis of 137 patients who underwent quantitative assessment by ${ }^{18} \mathrm{~F}$-FDG PET imaging for suspected CS. Pathologic right ventricular ${ }^{18} \mathrm{~F}-\mathrm{FDG}$ uptake was more common in patients with cardiovascular events than in those without events (46\% vs. $6 \%$ ), and a total cardiac metabolic activity value of more than $900 \mathrm{MBq}$ significantly predicted cardiac events (27\% vs. $4 \%$ ). Patients with pathologic right ventricular uptake had significantly higher total cardiac metabolic activity than those without right ventricular uptake. Therefore, the combination of pathologic right ventricular uptake and high total cardiac metabolic activity should be considered a significant risk factor in patients with CS $(43,44)$.

Cardiac PET is the preferred method for determining response to immunosuppressive therapy. Serial PET studies can be compared by visual or quantitative analysis of myocardial ${ }^{18}$ F-FDG uptake, with the latter being a more precise method to assess treatment response $(45,46)$. In one series, Osborne et al. (47) analyzed 23 patients who underwent serial PET examinations during immunosuppressive therapy for CS and found that a reduction in SUV intensity $\left(\mathrm{SUV}_{\max }\right)$ or extent (volume of inflammation above a prespecified SUV threshold) was associated with an improvement in left ventricular ejection fraction, whereas nonresponders to therapy (identified by changes in ${ }^{18} \mathrm{~F}$-FDG uptake) had a significant decrease in left ventricular ejection fraction. It has not been well established whether a change in ${ }^{18}$ F-FDG uptake is associated with a reduction in event rates. Further research is needed to identify appropriate and clinically meaningful quantitative techniques that can be compared longitudinally and to determine whether any other imaging techniques can be used to follow response to therapy.

\section{CMR}

The presence of LGE on delayed CMR imaging appears to be a strong prognosticator regarding future cardiac events and death in patients with CS $(39,42)$ and should be considered in clinical decision making on cardioverter-defibrillator implantation (9).

In a study by Kouranos et al. (19), 321 patients with extracardiac biopsy-proven sarcoidosis were followed over a median of 84 mo. LGE was the only independent predictor of the primary combined endpoint of all-cause mortality, sustained ventricular tachycardia episodes, or hospitalization for heart failure (hazard ratio, 5.68; $95 \%$ confidence interval, $1.74-18.49 ; P=0.004)$. CMR independently predicted adverse events (hazard ratio, 12.71; 95\% confidence interval, $1.48-109.35 ; P=0.021$ ) in patients with cardiac symptoms or abnormal electrocardiography findings.

Greulich et al. followed 155 patients with systemic sarcoidosis over a median of $2.6 \mathrm{y}$. LGE was present in 39 patients $(25.5 \%)$. The presence of LGE was the strongest independent risk factor (above left ventricular ejection fraction and end-diastolic volume), with a hazard ratio of 31.6 for death, aborted cardiac death, or appropriate implantable cardioverter-defibrillator discharge (12).

In the PET study by Tuominen et al., a subset of patients underwent CMR imaging; no adverse events were reported in patents who had no LGE, confirming an excellent negative predictive value of this finding (43).

In a metaanalysis by Hulten et al., 694 subject who underwent CMR were evaluated. The presence of LGE was associated with higher rates of death or ventricular arrhythmia (relative risk, 6.20; 95\% confidence interval, 2.47-15.6; $P<0.001$ ). In addition, the absence of LGE offered reassurance about the risk of ventricular arrhythmia or cardiovascular death (48).

In terms of prognosis, LGE appears to be more important than ${ }^{18}$ F-FDG uptake on PET imaging (49) and can therefore be used in decision making regarding device implantation.

\section{${ }^{18}$ F-FDG PET Versus CMR}

Several studies have compared the diagnostic capabilities of ${ }^{18} \mathrm{~F}$ FDG PET and CMR $(38,50,51)$. Interpretation of these studies requires caution for several reasons. First, most of the studies were underpowered to detect significant differences between these techniques. Second, the definition of gold standard in diagnosing CS remains elusive. Finally, these modalities detect different pathologic features of CS: inflammation with ${ }^{18} \mathrm{~F}$-FDG PET and largely scarring with LGE CMR. The ability of ${ }^{18}$ F-FDG PET to characterize inflammation can theoretically result in earlier diagnosis of CS (52). On the other hand, CMR has a higher spatial resolution than PET and can detect small areas of fibrosis. Although both ${ }^{18}$ F-FDG PET and CMR have high sensitivity, CMR may have a higher specificity in diagnosis of CS (53). Among patients who are already on steroid therapy, both CMR and PET may have reduced sensitivity but CMR may perform better (38). An important advantage of CMR is a substantially lower number of nondiagnostic scans than with ${ }^{18} \mathrm{~F}$ FDG PET, related to technical difficulties in patient preparation before the study. Additional benefits of CMR relative to ${ }^{18} \mathrm{~F}-\mathrm{FDG}$ PET include no exposure to ionizing radiation, no need for specialized patient preparation, and the ability to identify alternative cardiomyopathies or infiltrative diseases.

In a study by Ju Lee (51), 104 patients with suspected CS were evaluated by both CMR and ${ }^{18}$ F-FDG PET. LGE was seen in 79 $(76 \%)$ of the cases, compared with ${ }^{18} \mathrm{~F}$-FDG uptake being seen in only 31 cases $(30 \%)(P<0.001) .{ }^{18}$ F-FDG uptake without LGE was uncommon in that study. On the other hand, one small study (38) compared ${ }^{18}$ F-FDG PET with CMR and found that PET has a numerically higher sensitivity than CMR ( $88 \%$ vs. $77 \%$ ), although this difference was not statistically significant.

\section{Hybrid CMR/PET}

Although CMR and PET detect different histopathologic features of CS, the complementary role of these tests has not been well defined. Hybrid imaging with combined CMR/PET offers the advantage of an accurate assessment of function and identification of 


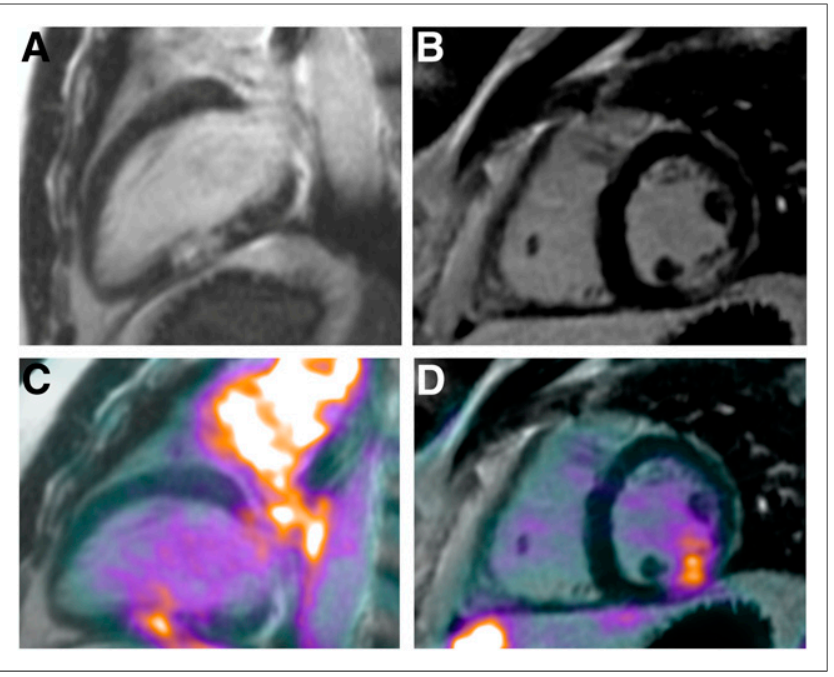

FIGURE 3. ${ }^{18} \mathrm{~F}-\mathrm{FDG}$ PET/CMR imaging in CMR-positive, PET-positive patient with CS. (A and B) Two-chamber (A) and short-axis (B) views demonstrate discrete area of LGE in inferolateral wall. (C and D). Fused ${ }^{18} \mathrm{~F}-\mathrm{FDG}$ PET/MR images show increased ${ }^{18} \mathrm{~F}$-FDG uptake in area of CMR abnormality

fibrosis by CMR, as well as assessment of inflammation using ${ }^{18} \mathrm{~F}$ FDG PET. Four patterns based on combined imaging results have been recognized. The first is CMR-positive, PET-positive, in which an LGE pattern is aligned with increased focal ${ }^{18} \mathrm{~F}$-FDG uptake, likely representing active CS (Fig. 3). The second pattern is CMR-positive, PET-negative, in which an LGE pattern is seen by CMR but no activity is detected by ${ }^{18} \mathrm{~F}-\mathrm{FDG}$, secondary to inactive CS with myocardial scarring (Fig. 4). The third pattern is CMR-negative, PETnegative, without LGE or ${ }^{18} \mathrm{~F}-\mathrm{FDG}$ uptake, likely a normal pattern. The fourth pattern is CMR-negative, PET-positive, in which LGE is negative but there is increased ${ }^{18} \mathrm{~F}-\mathrm{FDG}$ uptake (either focal, focalon-diffuse, or diffuse). This last pattern may signify a false-positive study due to failed myocardial suppression or physiologic uptake by normal myocardium, although in some cases this pattern may also represent an early stage of CS that is not yet visible on LGE. were evaluated by both CMR and PET. Overall, 91 patients $(85 \%)$ were LGE-positive, and among these 91 patients, 60 patients (66\%) had abnormal ${ }^{18}$ F-FDG uptake suggestive of active inflammation. In patients having both LGE and ${ }^{18} \mathrm{~F}-\mathrm{FDG}$ uptake, the likelihood of CS was higher, and a significantly higher use of immunosuppressive therapies was observed.
In a study by Vita et al. (54), 107 patients with suspected CS

A study by Dweck et al. evaluated 25 patients with hybrid PET and CMR imaging to detect active sarcoidosis, but this study featured simultaneous acquisition. ${ }^{18} \mathrm{~F}$-FDG uptake was quantified using the maximum target-to-normal myocardium ratio. These ratios were $50 \%$ higher in active CS (CMR-positive, PET-positive) than in inactive CS or false-positive studies (CMR-negative, PET-positive) (1.6 [interquartile range, 1.3-1.9] vs. 1.1 [interquartile range, 1.0-1.1], respectively; $P<0.001)$. The study also showed a high sensitivity and specificity for this hybrid approach to detect active CS and a positive correlation between T2 mapping and the target-to-normal myocardium ratios, although the correlation was not statistically significant. The optimal threshold for maximum target-to-normal myocardium ratio using the Youden index was 1.2, with a sensitivity of $100 \%$ and a specificity of $94 \%$, along with the benefit of lower radiation exposure $(8.2 \pm 1.5 \mathrm{mSv})$ than with ${ }^{18} \mathrm{~F}$-FDG PET studies, which often require a perfusion tracer for myocardial scar assessment (55).

In many patients with suspected CS, the combination of CMR and PET findings provides complementary value for diagnosis; assessment of myocardial function, pattern of injury, and disease activity; and management of CS (56). Integrating data from both imaging modalities can improve efficiency and reduce patient radiation exposure, with the benefit of providing a unifying approach for diagnosis and management of CS. Although simultaneous CMR/PET acquisition is a promising approach, the current availability of scanners capable of this type of hybrid imaging is very limited.

\section{CLINICAL USE OF ADVANCED CARDIAC IMAGING IN CS}

Advanced imaging modalities have been integrated into the clinical care of patients with suspected and established CS on the basis of the emerging data from multiple, mostly single-center studies. These studies largely reflect an institutional experience and suffer from a significant heterogeneity in clinical and imaging approaches. Although several publications have attempted to issue clinical recommendations based on the available evidence, these statements acknowledge limitations and challenges faced by the writing committees $(9,30,31)$. Advanced cardiac imaging appears to be an essential decision-assist tool in several clinical scenarios in patients with suspected or established CS, described below (Table 1).

\section{Diagnostic Tool in Patients with Established \\ Extracardiac Sarcoidosis}

Patients with biopsy-proven extracardiac sarcoidosis should be routinely screened for CS (10). The suggested initial screening includes careful history taking, electrocardiography, transthoracic echocardiography, and possibly 24-h Holter monitoring (9). Any abnormalities on the initial screening should be followed by advanced cardiac imaging.
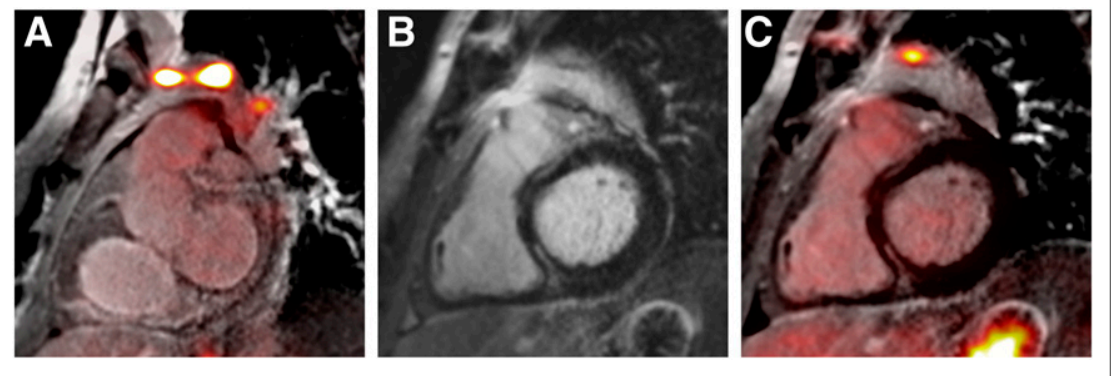

FIGURE 4. ${ }^{18} \mathrm{~F}$-FDG PET/CMR imaging in CMR-positive, PET-negative patient with CS. Shortaxis views demonstrate avid ${ }^{18} \mathrm{~F}$-FDG uptake in mediastinal lymph nodes on fused ${ }^{18} \mathrm{~F}$-FDG PET/ MR image (A) and discrete midmyocardial areas of LGE in antero- and inferoseptum (B) without matching increase in ${ }^{18} \mathrm{~F}-\mathrm{FDG}$ uptake (C). CMR is considered the initial test of choice; in patients with contraindications to CMR, ${ }^{18}$ F-FDG PET combined with myocardial perfusion imaging is an established diagnostic strategy $(30,31)$. Occasionally, ${ }^{18} \mathrm{~F}-\mathrm{FDG}$ PET is performed in patients with strongly suspected CS and negative CMR findings. CMR/PET hybrid imaging is also reasonable in institutions capable of this technique.

\section{Diagnostic Tool in Patients Without Established Extracardiac Sarcoidosis}

Isolated CS, an underdiagnosed entity, represents a great clinical challenge. There 


\begin{tabular}{lc}
\hline \multicolumn{1}{c}{ Clinical question } & Clinical context \\
\hline $\begin{array}{c}\text { Diagnosis of CS in patients with established extracardiac } \\
\text { sarcoidosis }\end{array}$ & $\begin{array}{c}\text { Positive initial screening: history, electrocardiogram, } \\
\text { echocardiogram, or 24-h Holter monitoring }\end{array}$ \\
\hline $\begin{array}{l}\text { Diagnosis of CS in patients without established } \\
\text { extracardiac sarcoidosis }\end{array}$ & $\begin{array}{c}\text { High degree of suspicion; examples include unexplained electrical } \\
\text { abnormalities and unexplained cardiomyopathy }\end{array}$ \\
\hline Initiation and monitoring of immunosuppressive therapy & $\begin{array}{c}\text { Identification and quantification of inflammation, including } \\
\text { longitudinal follow-up }\end{array}$ \\
\hline Device therapy/implantable cardioverter-defibrillator & $\begin{array}{c}\text { Identification of high-risk patients for sudden cardiac death beyond } \\
\text { traditional markers (left ventricular ejection fraction) }\end{array}$ \\
\hline
\end{tabular}

is no general consensus on when CS should be clinically suspected in patients without proven extracardiac sarcoidosis and what constitutes the most practical approach to diagnosis. One document suggests evaluation for CS in young patients $(<60 \mathrm{y}$ old $)$ with unexplained advanced atrioventricular block (9). Other clinical situations in which CS should be suspected include unexplained monomorphic ventricular tachycardia and unexplained cardiomyopathy in a young patient, especially with discrete regional wall motion abnormalities on echocardiography without coronary artery disease. The evaluation for possible CS should include CT of the chest, looking for evidence of extracardiac sarcoidosis, as well as advanced cardiac imaging (CMR or ${ }^{18}$ F-FDG PET). Suspected extracardiac lesions identified on chest imaging can be targets for biopsy. Unfortunately, cardiac imaging findings in patients without established extracardiac sarcoidosis can be nonspecific. In these cases, electroanatomic mapping or imaging-guided endomyocardial biopsy should be considered (17).

\section{Therapeutic Response Monitoring in Patients with Established Inflammatory CS}

Immunosuppressive therapy is commonly used in patients with CS, especially those with ventricular dysfunction and electrical abnormalities. Echocardiography and CMR imaging provide an overall assessment of ventricular function but cannot be used to reliably assess the inflammatory burden. T2-weighted imaging on CMR, although theoretically appealing, is not clinically used for therapeutic response monitoring. On the other hand, ${ }^{18}$ F-FDG PET has gained some observational evidence supporting its clinical utility in therapy initiation and therapeutic response monitoring. Unfortunately, there is limited evidence on optimal monitoring methods (descriptive or qualitative metrics), therapy-specific scan changes, and optimal monitoring intervals $(30,31)$.

\section{Prognostication in Patients with Established CS Considered for Device Therapy}

Advanced cardiac imaging has an important role in patients with established CS considered for an implantable cardioverter-defibrillator for primary prevention of sudden cardiac death. In patients with decreased left ventricular ejection fraction $(<35 \%)$, inflammation should be identified and treated. There is a strong indication for implantable cardioverter-defibrillator implantation in these patients if the systolic function does not improve with medical therapy and a trial of immunosuppression (in patients with inflammation). On the other hand, the presence of LGE in patients with mild to moderate left ventricular systolic dysfunction (ejection fraction, 35\%-49\%) or right ventricular dysfunction (ejection fraction, $<40 \%$ ) may also support cardioverter-defibrillator implantation (9).
The scenarios presented above describe common clinical approaches but also highlight uncertainties and knowledge gaps. It is imperative that future multicenter collaborative studies use systematic and uniform approaches to integration of advanced cardiac imaging into clinical decision making in patients with CS.

\section{CONCLUSION}

Advanced cardiac imaging techniques have improved the ability to diagnose CS, identify high-risk patients for cardiovascular events, and evaluate response to immunosuppressive therapy. Each imaging modality identifies certain histopathologic features of CS: LGE by delayed CMR imaging is useful for predominantly evaluating fibrosis, whereas PET is best suited for visualizing and quantifying active inflammation. CMR is an attractive initial testing option, and the presence of LGE is the strongest available prognostic marker in patients with CS. Cardiac PET is a good option if CMR is contraindicated and in occasional cases of strong clinical suspicion with normal CMR findings. Cardiac PET is best suited for patients receiving treatment for $\mathrm{CS}$ in determining response to immunosuppressive therapy. Hybrid imaging with CMR/PET is an exciting new imaging approach that incorporates the advantages of both imaging techniques with a single scan and thus may provide greater certainty in diagnosis of CS. Additional studies are needed to compare the findings of different imaging techniques to assess disease activity and to monitor therapeutic response.

\section{REFERENCES}

1. Iannuzzi MC, Rybicki BA, Teirstein AS. Sarcoidosis. N Engl J Med. 2007;357:21532165.

2. Perry A, Vuitch F. Causes of death in patients with sarcoidosis: a morphologic study of 38 autopsies with clinicopathologic correlations. Arch Pathol Lab Med. 1995; 119:167-172.

3. Gideon NM, Mannino DM. Sarcoidosis mortality in the United States, 1979-1991: an analysis of multiple-cause mortality data. Am J Med. 1996;100:423-427.

4. Cheong BY, Muthupillai R, Nemeth M, et al. The utility of delayed-enhancement magnetic resonance imaging for identifying nonischemic myocardial fibrosis in asymptomatic patients with biopsy-proven systemic sarcoidosis. Sarcoidosis Vasc Diffuse Lung Dis. 2009;26:39-46.

5. Freeman AM, Curran-Everett D, Weinberger HD, et al. Predictors of cardiac sarcoidosis using commonly available cardiac studies. Am J Cardiol. 2013;112:280-285.

6. Patel MR, Cawley PJ, Heitner JF, et al. Detection of myocardial damage in patients with sarcoidosis. Circulation. 2009;120:1969-1977.

7. Cooper LT, Baughman KL, Feldman AM, et al. The role of endomyocardial biopsy in the management of cardiovascular disease. Eur Heart J. 2007;28: 3076-3093.

8. Uemura A, Morimoto S, Hiramitsu S, Kato Y, Ito T, Hishida H. Histologic diagnostic rate of cardiac sarcoidosis: evaluation of endomyocardial biopsies. Am Heart J. 1999;138:299-302. 
9. Birnie DH, Sauer WH, Bogun F, et al. HRS expert consensus statement on the diagnosis and management of arrhythmias associated with cardiac sarcoidosis. Heart Rhythm. 2014;11:1305-1323.

10. Mehta D, Lubitz SA, Frankel Z, et al. Cardiac involvement in patients with sarcoidosis: diagnostic and prognostic value of outpatient testing. Chest. 2008;133:1426-1435.

11. Smedema JP, Snoep G, van Kroonenburgh MP, et al. Cardiac involvement in patients with pulmonary sarcoidosis assessed at two university medical centers in the Netherlands. Chest. 2005;128:30-35.

12. Greulich S, Deluigi CC, Gloekler S, et al. CMR imaging predicts death and other adverse events in suspected cardiac sarcoidosis. JACC Cardiovasc Imaging. 2013;6:501-511.

13. Blankstein R, Osborne M, Naya M, et al. Cardiac positron emission tomography enhances prognostic assessments of patients with suspected cardiac sarcoidosis. J Am Coll Cardiol. 2014;63:329-336.

14. Lagana SM, Parwani AV, Nichols L. Cardiac sarcoidosis: a pathology focused review. Arch Pathol Lab Med. 2010;134:1039-1046.

15. Sekhri V, Sanal S, Delorenzo LJ, Aronow WS, Maguire GP. Cardiac sarcoidosis: a comprehensive review. Arch Med Sci. 2011;7:546-554.

16. Tavora F, Cresswell N, Li L, Ripple M, Solomon C, Burke A. Comparison of necropsy findings in patients with sarcoidosis dying suddenly from cardiac sarcoidosis versus dying suddenly from other causes. Am J Cardiol. 2009; 104:571-577.

17. Liang JJ, Hebl VB, DeSimone CV, et al. Electrogram-guidance: a method to increase the precision and diagnostic yield of endomyocardial biopsy for suspected cardiac sarcoidosis and myocarditis. JACC Heart Fail. 2014;2:466-473.

18. Burstow DJ, Tajik AJ, Bailey KR, et al. Two-dimensional echocardiographic findings in systemic sarcoidosis. Am J Cardiol. 1989;63:478-482.

19. Kouranos V, Tzelepis G, Rapti A, et al. Complementary role of CMR to conventional screening in the diagnosis and prognosis of cardiac sarcoidosis. JACC Cardiovasc Imaging . 2017;10:1437-1447.

20. Nagano N, Nagai T, Sugano Y, et al. Association between basal thinning of interventricular septum and adverse long-term clinical outcomes in patients with cardiac sarcoidosis. Circ J. 2015;79:1601-1608.

21. Rapti A, Kouranos V, Gialafos E, et al. Elevated pulmonary arterial systolic pressure in patients with sarcoidosis: prevalence and risk factors. Lung. 2013;191:61-67.

22. Nunes H, Uzunhan Y, Freynet O, et al. Pulmonary hypertension complicating sarcoidosis. Presse Med. 2012;41:e303-316.

23. Joyce E, Ninaber MK, Katsanos S, et al. Subclinical left ventricular dysfunction by echocardiographic speckle-tracking strain analysis relates to outcome in sarcoidosis. Eur J Heart Fail. 2015;17:51-62.

24. Eguchi M, Tsuchihashi K, Hotta D, et al. Technetium-99m sestamibi/tetrofosmin myocardial perfusion scanning in cardiac and noncardiac sarcoidosis. Cardiology. 2000;94:193-199.

25. Le Guludec D, Menad F, Faraggi M, Weinmann P, Battesti J-P, Valeyre D. Myocardial sarcoidosis: clinical value of technetium-99m sestamibi tomoscintigraphy. Chest. 1994;106:1675-1682.

26. Surasi DS, Manapragada PP, Lloyd SG, Bhambhvani P. Role of multimodality imaging including thallium-201 myocardial perfusion imaging in the diagnosis and monitoring of treatment response in cardiac sarcoidosis. $\mathrm{J} \mathrm{Nucl} \mathrm{Cardiol.}$ 2014;21:849-852.

27. Futamatsu H, Suzuki J, Adachi S, et al. Utility of gallium-67 scintigraphy for evaluation of cardiac sarcoidosis with ventricular tachycardia. Int $J$ Cardiovasc Imaging. 2006;22:443-448.

28. Silberstein EB, DeVries DF. Reverse redistribution phenomenon in thallium-201 stress tests: angiographic correlation and clinical significance. J Nucl Med. 1985;26:707-710.

29. Hirose Y, Ishida Y, Hayashida K, et al. Myocardial involvement in patients with sarcoidosis: an analysis of 75 patients. Clin Nucl Med. 1994;19:522526.

30. Chareonthaitawee P, Beanlands RS, Chen W, et al. Joint SNMMI-ASNC expert consensus document on the role of ${ }^{18} \mathrm{~F}$-FDG PET/CT in cardiac sarcoid detection and therapy monitoring. J Nucl Cardiol. 2017;24:1741-1758.

31. Slart RHJA, Glaudemans AWJM, Lancellotti P, et al. A joint procedural position statement on imaging in cardiac sarcoidosis: from the Cardiovascular and Inflammation \& Infection Committees of the European Association of Nuclear Medicine, the European Association of Cardiovascular Imaging, and the American Society of Nuclear Cardiology. Eur Heart J Cardiovasc Imaging. 2017;18: 1073-1089.

32. Youssef G, Leung E, Mylonas I, et al. The use of ${ }^{18}$ F-FDG PET in the diagnosis of cardiac sarcoidosis: a systematic review and metaanalysis including the Ontario experience. J Nucl Med. 2012;53:241-248.
33. Kim SJ, Pak K, Kim K. Diagnostic performance of F-18 FDG PET for detection of cardiac sarcoidosis: a systematic review and meta-analysis. J Nucl Cardiol 2019;1:1-13.

34. Osborne MT, Hulten EA, Murthy VL, et al. Patient preparation for cardiac fluorine-18 fluorodeoxyglucose positron emission tomography imaging of inflammation. J Nucl Cardiol. 2017;24:86-99.

35. Norikane T, Yamamoto Y, Maeda Y, et al. Comparative evaluation of ${ }^{18} \mathrm{~F}-\mathrm{FLT}$ and ${ }^{18} \mathrm{~F}$-FDG for detecting cardiac and extra-cardiac thoracic involvement in patients with newly diagnosed sarcoidosis. EJNMMI Res. 2017;7:69.

36. Lapa C, Reiter T, Kircher M, et al. Somatostatin receptor based PET/CT in patients with the suspicion of cardiac sarcoidosis: an initial comparison to cardiac MRI. Oncotarget. 2016;7:77807-77814.

37. Gormsen LC, Haraldsen A, Kramner S, et al. A dual tracer ${ }^{68} \mathrm{Ga}-\mathrm{DOTANOC}$ PET/CT and ${ }^{18} \mathrm{~F}-\mathrm{FDG}$ PET/CT pilot study for detection of cardiac sarcoidosis. EJNMMI Res. 2016;6:52.

38. Ohira H, Tsujino I, Ishimaru S, et al. Myocardial imaging with ${ }^{18} \mathrm{~F}$-fluoro-2deoxyglucose positron emission tomography and magnetic resonance imaging in sarcoidosis. Eur J Nucl Med Mol Imaging. 2008;35:933-941.

39. Smedema JP, Snoep G, van Kroonenburgh MP, et al. Evaluation of the accuracy of gadolinium-enhanced cardiovascular magnetic resonance in the diagnosis of cardiac sarcoidosis. J Am Coll Cardiol. 2005;45:1683-1690.

40. Crouser ED, Ono C, Tran T, He X, Raman SV. Improved detection of cardiac sarcoidosis using magnetic resonance with myocardial T2 mapping. Am J Respir Crit Care Med. 2014;189:109-112.

41. Amano Y, Tachi M, Tani H, Mizuno K, Kobayashi Y, Kumita S. T2-weighted cardiac magnetic resonance imaging of edema in myocardial diseases. ScientificWorldJournal. 2012;2012:194069.

42. Ichinose A, Otani H, Oikawa M, et al. MRI of cardiac sarcoidosis: basal and subepicardial localization of myocardial lesions and their effect on left ventricular function. AJR. 2008;191:862-869.

43. Tuominen H, Haarala A, Tikkakoski A, et al. FDG-PET in possible cardiac sarcoidosis: right ventricular uptake and high total cardiac metabolic activity predict cardiovascular events. J Nucl Cardiol. 2019;2:1-7.

44. Laudicella R, Minutoli F, Baldari S. Prognostic insights of molecular imaging in cardiac sarcoidosis. J Nucl Cardiol. 2019;4:1-3.

45. Ahmadian A, Brogan A, Berman J, et al. Quantitative interpretation of FDG PET/CT with myocardial perfusion imaging increases diagnostic information in the evaluation of cardiac sarcoidosis. J Nucl Cardiol. 2014;21:925-939.

46. Waller AH, Blankstein R. Quantifying myocardial inflammation using F18-fluorodeoxyglucose positron emission tomography in cardiac sarcoidosis. $\mathrm{J} \mathrm{Nucl}$ Cardiol. 2014;21:940-943.

47. Osborne MT, Hulten EA, Singh A, et al. Reduction in ${ }^{18} \mathrm{~F}$-fluorodeoxyglucose uptake on serial cardiac positron emission tomography is associated with improved left ventricular ejection fraction in patients with cardiac sarcoidosis. $J$ Nucl Cardiol. 2014;21:166-174.

48. Hulten E, Agarwal V, Cahill M, et al. Presence of late gadolinium enhancement by cardiac magnetic resonance among patients with suspected cardiac sarcoidosis is associated with adverse cardiovascular prognosis: a systematic review and meta-analysis. Circ Cardiovasc Imaging. 2016;9:e005001.

49. Bravo PE, Raghu G, Rosenthal DG, et al. Risk assessment of patients with clinical manifestations of cardiac sarcoidosis with positron emission tomography and magnetic resonance imaging. Int J Cardiol. 2017;241:457-462.

50. Orii M, Hirata K, Tanimoto T, et al. Comparison of cardiac MRI and ${ }^{18} \mathrm{~F}-\mathrm{FDG}$ positron emission tomography manifestations and regional response to corticosteroid therapy in newly diagnosed cardiac sarcoidosis with complete heart block. Heart Rhythm. 2015;12:2477-2485.

51. Ju Lee N, Lee B, Litt H. Cardiac MRI vs. myocardial ${ }^{18} \mathrm{~F}-\mathrm{FDG}$ PET/CT in patients with clinical concern for cardiac sarcoid [abstract]. J Cardiovasc Magn Reson. 2015;17(suppl 1):O30.

52. Skali H, Schulman AR, Dorbala S. ${ }^{18}$ F-FDG PET/CT for the assessment of myocardial sarcoidosis. Curr Cardiol Rep. 2013;15:352.

53. Aggarwal NR, Snipelisky D, Young PM, Gersh BJ, Cooper LT, Chareonthaitawee P. Advances in imaging for diagnosis and management of cardiac sarcoidosis. Eur Heart J Cardiovasc Imaging. 2015;16:949-958.

54. Vita T, Okada DR, Veillet-Chowdhury M, et al. Complementary value of cardiac magnetic resonance imaging and positron emission tomography/computed tomography in the assessment of cardiac sarcoidosis. Circ Cardiovasc Imaging. 2018;11:e007030.

55. Dweck MR, Abgral R, Trivieri MG, et al. Hybrid magnetic resonance imaging and positron emission tomography with fluorodeoxyglucose to diagnose active cardiac sarcoidosis. JACC Cardiovasc Imaging. 2018;11:94-107.

56. Ahmadian A, Pawar S, Govender P, Berman J, Ruberg FL, Miller EJ. The response of FDG uptake to immunosuppressive treatment on FDG PET/CT imaging for cardiac sarcoidosis. J Nucl Cardiol. 2017;24:413-424. 